
RAPPORTO AI SEQUESTRI E DISTRUZIONI EFFETTUATI IN UN MACELLO DELL'ITALIA CENTRALE NEL PERIODO 2004-2009

\title{
CONSIDERATIONS ON MEAT INSPECTION PROCEDURES INTHE LIGHT OF CONDEMNATIONS AT AN ITALIAN EU SLAUGHTERHOUSE IN THE PERIOD 2004-2009
}

\author{
Garofalo D. ${ }^{1}$, Budelli L. ${ }^{2}$, Cambiotti V. ${ }^{2}$, Cenci Goga B.T. ${ }^{2}$ \\ ${ }^{1}$ Master in Sanità Pubblica Veterinaria e Igiene degli Alimenti, Università degli Studi di Perugia \\ ${ }^{2}$ U.S.L. n.2, Perugia, Dipartimento di Prevenzione, Servizio Veterinario di Igiene degli Alimenti di \\ Origine Animale
}

\section{SUMMARY}

KEYWORDS

\begin{abstract}
Post-mortem findings of 373.901 cattle slaughtered at an EC abattoir are reported. Results show high incidence of pneumonia and hepatic lesions. Total condemnation of the whole carcass has happened 91 times in Bovine species, 1073 times in swine species, 40 in sheep. In the light of the EC Regulation N.854/2004 of the European parliament and of the council of 29th April 2004 that lays down specific rules for the organization of official controls of products of animal origin destined for human consumption, authors outline the examples of simplification and partial redrafting of the existing laws on the basis of the opinions expresses by the EFSA. Comparison to other European countries and an estimate of economic damage are discussed.
\end{abstract}

post, mortem, inspection, EFSA, condemnation

\section{INTRODUZIONE}

In previsione delle modifiche legislative comunitarie delle indicazioni per la riformulazione e semplificazione della normativa vigente, (Regolamenti 852, 853 e 854 del 2004) all'EFSA (European Food Safety Authority) sono stati posti dei quesiti specifici sulle metodiche dell'ispezione post-mortem (1).

In particolare, l'EFSA è stata chiamata a definire, per ciascuna delle procedure di palpazione ed incisione precedentemente obbligatorie, in ottemperanza alla Direttiva del Consiglio 64/433 (modificata e codificata dalla Direttiva del Consiglio 91/497, attuata nel nostro paese con Decreto Legislativo n. 286 del 1994), le seguenti condizioni: i) quali malattie o condizioni patologiche sono evidenziate, ii) l'agente patogeno e la sua pericolosità per l'uomo, iii) i rischi per la salute pubblica, qualora determinate pro- cedure (nella fattispecie la palpazione e l'incisione) siano omesse nel corso dell'ispezione post-mortem di animai allevati nell'ambito di sistemi integrati di produzione, iv) la possibilità che metodi alternativi di laboratorio o test rapidi possano garantire un livello diagnostico e di tutela della salute pubblica almeno equivalente da quello offerto dalle procedure correnti (2). Scopo del presente lavoro è quello di analizzare le cause di sequestro e distruzione effettuati in un macello dell'Italia centrale nel periodo 20042009 alla luce dei pareri espressi dall'EFSA (3). Nel lavoro è presente una stima del danno economico.

\section{MATERIALI E METODI}

Sono stati esaminati i sequestri parziali o totali di organi di ungulati (bovini, ovini, suini) regolarmente macellati negli anni 2004-2009 presso un pubblico macello CEE della provincia di Pe- 
rugia con capacita di macellazione annua di 10.000 UGB. Precisamente i sequestri parziali sono stati di 10903 a carico di bovini, 3195 per gli ovini e 25903 sono gli organi di suini sequestrati. Mentre le carcasse a cui è stato imposto il sequestro e la distruzione sono state nel periodo in esame di 91 carcasse di bovini, 40 carcasse di ovini e 1073 di suini. Per ciascun animale venivano annotate su apposita scheda, le alterazioni riscontrate alla visita post-mortem che comportavano il sequestro totale o parziale. Il trasferimento dei dati su computer è stato effettuato con il software Microsoft Excel per Windows 2010, Inc. I file, salvati sottoforma di foglio elettronico .xls sono stati importati su FileMaker pro 10.0v1, Inc. per la elaborazione dei dati. Inoltre, il software è stato utilizzato per il calcolo dei costi dovuti agli scarti. Il prezzo della carne nel periodo in esame è stato indicato dalla Camera di Commercio di Perugia.

\section{RISULTATI}

I risultati della visita post-mortem relativi al totale delle alterazioni riscontrate nei vari organi della specie bovina sono riportati nelle tabelle 1 e 2 . Molti soggetti hanno presentato alterazioni a carico di più organi, ma, nel complesso, il polmone è stato l'organo più colpito con $51,15 \%$ dei sequestri parziali nella specie bovina. Nelle tabelle 1 e 2 nelle ultime due colonne, sono riportate le cause di sequestro totale della carcassa, avvenuto 91 volte nella specie bovina. Le cause più frequenti sono state nefriti, DFD e mancata acidificazione delle carni, polmoniti. La distribuzione delle lesioni polmonari appare omogenea ma con maggiore prevalenza di pleuriti rispetto a polmoniti e broncopolmoniti. Altro dato interessante appare l'elevata prevalenza della distomatosi e dell'epatite apostematosa, nonché della echinococcosi/idatidiosi che testimonia la presenza della malattia nella specie canina. La tubercolosi bovina è stata diagnosticata 19 volte come complesso primario $(0.17 \%)$ e mai in forma generalizzata. La cisticercosi è stata causa di 5 sequestri parziali nei suini $(0.02 \%)$ e mai diagnosticata nei bovini. La stima della perdita economica relativa alla carne sequestrata è di $92.976 €$ per la carne bovina, $160.446 €$ per la carne suina, $2511 €$ per la carne ovina.

\section{CONSIDERAZIONI E CONCLUSIONI}

Dall'esame dei risultati emerge la presenza di lesioni che rientrano in una gamma piuttosto ristretta e prevalentemente localizzate al polmone e al fegato. Va pertanto affermata l'importanza della raccolta dei dati, vale a dire la registrazione di tutte le alterazioni evidenziate in sede di macellazione sia per attivare un flusso di informazioni dal mattatoio all'allevamento, utile anche alla prevenzione delle malattie esistenti, sia per fare assumere pienamente al mattatoio il ruolo di osservatorio epidemiologico più volte richiamato e mai completamente realizzato. Il Reg. (CE) N. 2074/2005 della commissione recante modalità di attuazione relative a taluni punti del regolamento (CE) n. 853/2004 descrive come devono essere comunicate le informazioni sulla catena alimentare, nonché i risultati delle ispezioni ante e post-mortem cioè il flusso di dati tra allevamento e mattatoio. Suggerisce tra l'altro l'uso di modelli e codici. Se è vero che gli animali possono andare incontro a infezioni causate da agenti patogeni che provocano la comparsa di segni clinici, rilevabili nel corso dell'ispezione ante-mortem e di lesioni individuabili durante l'ispezione post-mortem, la letteratura scientifica indica che l'ispezione post-mortem di animali apparentemente sani permette di rilevare soltanto il $20 \%$ di tutte le lesioni macroscopiche e che tali lesioni sono presenti in una percentuale pari o inferiore all'1\% degli animali macellati (4). Inoltre, la maggior parte delle lesioni macroscopiche evidenziate all'esame anatomopatologico permette di diagnosticare anche malattie degli animali che non hanno risvolto sulla salute pubblica. È generalmente accettato dalla comunità scientifica che l'efficacia della routine dell'ispezione post-mortem per il rilievo di condizioni e di lesioni con risvolti sulla salute pubblica è limitata (5). Il Regolamento N. 854/2004 del Parlamento Europeo e del Consiglio del 29 aprile 2004, che stabilisce norme specifiche per l'organizzazione di controlli ufficiali sui prodotti di origine animale destinati al consumo umano è un primo esempio, sebbene moderato, di semplificazione e di riformulazione delle norme esistenti effettuate sulla base dei pareri dell'EFSA.(6) L'EFSA apre le porte ad uno scenario futuro dove l'ispezione post-mortem sarà affiancata da nuove tecniche diagnostiche per limitare le contaminazioni crociate. Sarà possibile distinguere tra animali a rischio e non a rischio grazie alla ricerca delle proteine della fase acuta (APP) o all'uso di analisi di immagini computerizzate e si potrà valutare all'istante la carica microbica grazie a metodi che ricercano componenti chimiche legate ai microrganismi (rapid m-ATP assay) (3) o la contaminazione fecale grazie alla ricerca della clorofilla (VerifEYE chlorofill) (3). 
Tabella 1. Lesioni e motivazioni per cui è stato imposto il sequestro e distruzione di organi e carcasse di Bovini nel periodo 2004-2009.

\begin{tabular}{|l|c|c|c|c|}
\hline \multicolumn{1}{|c|}{$\begin{array}{c}\text { diagnosi } \\
\text { Bovini }\end{array}$} & $\begin{array}{c}\mathbf{n}^{\circ} \\
\text { organi }\end{array}$ & $\%$ & $\begin{array}{c}\mathbf{n}^{\circ} \\
\text { carcasse } \\
\text { seq. }\end{array}$ & $\%$ \\
\hline Ascessi diffusi & 1135 & 10,41 & 6 & 6,59 \\
\hline Distomatosi & 804 & 7,37 & 0 & 0,00 \\
\hline Dicroceliosi & 356 & 3,27 & 0 & 0,00 \\
\hline $\begin{array}{l}\text { Contengono un } \\
\text { corpo estaneo }\end{array}$ & 324 & 2,97 & 0 & 0,00 \\
\hline Parassitosi & 164 & 1,50 & 0 & 0,00 \\
\hline $\begin{array}{l}\text { Echinococcosi } \\
\text { /Idatidiosi }\end{array}$ & 88 & 0,81 & 0 & 0,00 \\
\hline $\begin{array}{l}\text { Inalazzione } \\
\text { ematica }\end{array}$ & 48 & 0,44 & 0 & 0,00 \\
\hline Polisierosite & 36 & 0,33 & 0 & 0,00 \\
\hline Fascioliasi & 35 & 0,32 & 0 & 0,00 \\
\hline Ascaridiosi & 33 & 0,30 & 0 & 0,00 \\
\hline Tubercolosi & 19 & 0,17 & 0 & 0,00 \\
\hline Actinomicosi & 9 & 0,08 & 0 & 0,00 \\
\hline Strongiliosi & 5 & 0,05 & 0 & 0,00 \\
\hline Imbrattate & 3 & 0,03 & 0 & 0,00 \\
\hline Actinobacillosi & 2 & 0,02 & 0 & 0,00 \\
\hline $\begin{array}{l}\text { DFD e } \\
\text { mancata } \\
\text { acidificazione } \\
\text { delle carni }\end{array}$ & 0 & 0,00 & 8 & 8,79 \\
\hline $\begin{array}{l}\text { Non corretta } \\
\text { identificazione }\end{array}$ & 0 & 0,00 & 7 & 7,69 \\
\hline $\begin{array}{l}\text { morti prima } \\
\text { della } \\
\text { macellazione }\end{array}$ & 0 & 0,00 & 6 & 6,59 \\
\hline $\begin{array}{l}\text { Putrefazione e } \\
\text { ammuffimento }\end{array}$ & 0 & 0,00 & 6 & 6,59 \\
\hline Setticemia & 0 & 0,00 & 5 & 5,49 \\
\hline Cachessia & 0 & 0,00 & 3 & 3,30 \\
\hline Linfoadenite & 0 & 0,00 & 2 & 2,20 \\
\hline ittero & 0 & 0,00 & 2 & 2,20 \\
\hline Sarcosporidiosi & 0 & 0,00 & 2 & 2,20 \\
\hline Peritonite & 0 & 0,00 & 16 & 17,58 \\
\hline
\end{tabular}

Tabella 2. Lesioni e motivazioni per cui è stato imposto il sequestro e distruzione di organi e carcasse di Bovini nel periodo 2004-2009

\begin{tabular}{|l|c|c|c|c|}
\hline \multicolumn{1}{|c|}{$\begin{array}{c}\text { Lesioni } \\
\text { Bovini }\end{array}$} & $\begin{array}{c}\mathbf{n}^{\circ} \\
\text { organi }\end{array}$ & $\%$ & $\begin{array}{c}\mathbf{n}^{\circ} \\
\text { carcasse } \\
\text { seq. }\end{array}$ & $\%$ \\
\hline Pleurite & 2689 & 24,66 & 0 & 0,00 \\
\hline Polmonite & 1182 & 10,84 & 8 & 8,79 \\
\hline $\begin{array}{l}\text { Nefrite } \\
\text { cronica }\end{array}$ & 533 & 4,89 & 0 & 0,00 \\
\hline $\begin{array}{l}\text { Bronco- } \\
\text { polmonite }\end{array}$ & 525 & 4,82 & 0 & 0,00 \\
\hline Epatite & 159 & 1,46 & 0 & 0,00 \\
\hline $\begin{array}{l}\text { Polmonit.a } \\
\text { macchie } \\
\text { verdi }\end{array}$ & 145 & 1,33 & 0 & 0,00 \\
\hline $\begin{array}{l}\text { Enfisema } \\
\text { acuto }\end{array}$ & 136 & 1,25 & 3 & 3,30 \\
\hline
\end{tabular}

\begin{tabular}{|l|c|c|c|r|}
\hline Pericardite & 129 & 1,18 & 4 & 4,40 \\
\hline $\begin{array}{l}\text { Nefrite } \\
\text { interstiziale }\end{array}$ & 123 & 1,13 & 0 & 0,00 \\
\hline Periepatite & 110 & 1,01 & 0 & 0,00 \\
\hline $\begin{array}{l}\text { Enfisema } \\
\text { cronico }\end{array}$ & 104 & 0,95 & 0 & 0,00 \\
\hline $\begin{array}{l}\text { Calcolosi } \\
\text { epatica }\end{array}$ & 56 & 0,51 & 0 & 0,00 \\
\hline Epatosi & 52 & 0,48 & 1 & 1,10 \\
\hline Ruminite & 49 & 0,45 & 1 & 1,10 \\
\hline Melanosi & 49 & 0,45 & 0 & 0,00 \\
\hline Sierosite & 47 & 0,43 & 0 & 0,00 \\
\hline Steatosi & 46 & 0,42 & 2 & 2,20 \\
\hline Nefrite & 31 & 0,28 & 13 & 14,29 \\
\hline Nefrosi & 23 & 0,21 & 0 & 0,00 \\
\hline Idronefrosi & 11 & 0,10 & 0 & 0,00 \\
\hline $\begin{array}{l}\text { Trauma } 0 \\
\text { frattura }\end{array}$ & 10 & 0,09 & 4 & 4,40 \\
\hline $\begin{array}{l}\text { Infiltrazioni } \\
\text { siero } \\
\text { ematiche }\end{array}$ & 9 & 0,08 & 2 & 2,20 \\
\hline Cirrosi & 8 & 0,07 & 2 & 2,20 \\
\hline Splenite & 5 & 0,05 & 1 & 1,10 \\
\hline
\end{tabular}

\section{BIBLIOGRAFIA}

1. Cenci Goga B.T, (2004). Opinion of the Scientific Panel on Biological Hazards on Revision of Meat Inspection for Beef raised in Integrated Production Systems. The EFSA Journal 141, 1-48.

2. Cenci Goga B.T., (2007). Considerazioni sulla semplificazione dei metodi di ispezione post-mortem in rapporto ai sequestri e distruzioni effettuati in un macello dell'Italia centrale nel periodo 2000-2004. Atti del convegno SISVET 18/03/2007 2.

3. Cenci Goga B.T., (2004). Opinion of the Scientific Panel on Biological Hazards on the request from the Commission on Meat Inspection Procedures for Lambs and Goats. The EFSA Journal 54, 1-45.

4. Berends B.R., (1993). Efficacy of current EC meat inspection procedures and some proposed revisions with respect to microbiological safety: a critical review. Veterinary Record 133, 411-415.

5. Hathaway S.C., (1989). Impact of ovine meat inspection systems on processing and production costs. Veterinary Record 124, 189-193.

6. Cenci Goga B.T., (2005). Recenti attività dei gruppi di lavoro di esperti dell'EFSA sulla revisione dei metodi di ispezione delle carni. Il Progresso Veterinario 6, 245-247. 